\title{
Treatment Patterns and Outcomes Before and After Humulin R U-500 Initiation Among US Patients with Type 2 Diabetes Previously Prescribed $\leq 200$ Units/day of U-100 Insulin
}

Jieling Chen · Sujana Borra • Ahong Huang · Ludi Fan •

Roy Daniel Pollom · Robert C. Hood

Received: October 5, 2021 / Accepted: January 21, 2022 / Published online: February 21, 2022

(C) The Author(s) 2022

\section{ABSTRACT}

Introduction: Humulin R U-500 (U-500R) utilization has increased in the past few years, raising concerns as U-500R is indicated only for patients requiring $>200$ units of insulin. Thus, evidence of dispensed total daily dose (dTDD) > 200 units of prior U-100 insulin based on pharmacy claims is increasingly used as a criterion to determine appropriate switching to U-500R by payers. The study compared the treatment patterns and outcomes before and after U-500R initiation among patients who were identified with $\leq 200$ units/day U-100 insulin fill in order to understand the appropriateness of switching.

Methods: Patients with type 2 diabetes who initiated U-500R (index date $=$ first fill) with $\leq 200$ units/day pre-index dTDD

Supplementary Information The online version contains supplementary material available at https:// doi.org/10.1007/s13300-022-01209-z.

J. Chen $(\bowtie) \cdot$ L. Fan · R. D. Pollom

Eli Lilly and Company, Lilly Corporate Center, Indianapolis, IN 46285, USA

e-mail:whjane@gmail.com

S. Borra · A. Huang

STATinMED Research, Plano, TX, USA

R. C. Hood

Endocrine Clinic of Southeast Texas, Beaumont, TX, USA and $>200$ units/day post-index dTDD were identified in a Veterans Health Administration dataset between 1 January 2014 and 30 June 2017. Descriptive analysis was conducted on treatment patterns (dTDD, insulin dosage [units/kg], adherence, number of prescription fills) and clinical outcomes (HbA1c, symptomatic hypoglycemic events). Associations between U-500R exposure and outcomes were evaluated using mixed-effects models. Subgroups of U-500R syringe and KwikPen users were analyzed separately.

Results: Among 1191 U-500R initiators identified in the study the mean dTDD increased from the pre- to post-index periods ( 147.2 vs 346.3 ; $p<0.0001)$. The mean HbA1c decreased from pre- to post-initiation $(9.6 \%$ vs $8.6 \%$; $p<0.0001$ ), and symptomatic hypoglycemia events per patient per year increased (2.0 vs 3.3, $p<0.0001)$. Mixed-effects models confirmed the significance of the changes $(p<0.0001)$. Device subgroups followed similar trends.

Conclusions: U-500R initiation was associated with large dTDD increases, improved glycemic control, and modest increases in hypoglycemia events, suggesting U-500R initiation may have corrected previous treatment compliance issues. Imposing dTDDs $>200$ units before switching to U-500R criterion could hurt the opportunities for patients who need a simplified regimen for better outcomes. 
Keywords: Type 2 diabetes; Concentrated insulin; Humulin R U-500; Glycemic control

\section{Key Summary Points}

What is already known about this subject

U-500R is a concentrated insulin with simplified TID or BID regimens.

$\mathrm{U}-500 \mathrm{R}$ is an insulin monotherapy indicated for patients with diabetes mellitus requiring more than 200 units of insulin per day.

U-500R improves glycemic control effectively and safely with fewer injections in patients with T2D treated with highdose/high-volume U-100 insulin.

\section{What this study adds}

A significant portion of U-500R initiators were found to fill $\leq 200$ units/day U-100 insulin before switching and were associated with poor glycemic control.

U-500R initiation was associated with large increases in purchased insulin dosage as well as clinically important improvement in glycemic control, together with relatively modest increases in hypoglycemic events, suggesting U-500R addresses unmet patient insulin needs.

Insulin dose calculated on the basis of claims alone is not the same as a patient's insulin requirement; careful consideration of other factors is necessary to identify patients with high insulin needs and compliance issues in the real-world setting.

\section{INTRODUCTION}

Many patients with type 2 diabetes (T2D) who require high insulin doses ( $>200$ units total daily dose $[\mathrm{TDD}])$ face treatment compliance challenges that hinder their blood glucose management $[1,2]$. This is particularly concerning for patients prescribed standard insulin formulations such as U-100 [3, 4]. Compliance barriers among this patient population include treatment regimen-related distress, which may be caused by the complexity and inconvenience of multiple injections (with an average of approx. five per day) and multiple insulins, the increased need for glucose monitoring, and high injection volumes that cause injection site discomfort and pain [1, 3-5]. These barriers may contribute to noncompliance manifested through inconsistent injection self-administration or injection volume reduction, which in turn may result in serious suboptimal dosing $[1,4,5]$. These compliance barriers have been directly associated with poor glycemic control, which are further associated with adverse clinical and economic outcomes [2,6].

Humulin $\mathrm{R}^{\circledR}$ U-500 ([U-500R]; Eli Lilly \& Company, Indianapolis, IN, USA) is a concentrated insulin indicated for patients with diabetes who have high insulin requirements. In 2016, the US Food and Drug Administration (FDA) approved a dedicated U-500R syringe and the prefilled, disposable U-500R KwikPen ${ }^{\circledR}$ $[7,8]$. These new devices have allayed dosing error concerns and expanded clinician options for addressing variable individual dosing needs [9]. U-500R has unique basal/bolus properties suitable as a monotherapy, with simplified twice- and thrice-daily injection regimens [10-12]. With one-fifth the injection volume of U-100, the simplified dual basal/bolus U-500R insulin regimen significantly decreases treatment complexity, addresses injection discomfort, reduces the number of pen changes, and facilitates delivery of larger doses [13-15]. Clinical studies on high-dose insulin patients who switched to U-500R have shown significant improvement in glycemic control and patientreported outcomes, especially regarding treatment burden and disease management $[3,5,6,16,17]$. Likewise, patients initiating U-500R have shown significant improvement in patient-reported treatment burden and disease management outcomes [16-18].

The rise in U-500R utilization has sparked clinical and cost concerns among payers. Since 
U-500R is indicated for patients with high dose needs (requiring $>200$ units/day), payers are particularly concerned with a significant portion of patients switching from $\leq 200$ units/day of U-100 insulin regimens to U-500R [19]. Therefore, demonstrating insulin needs with the evidence of $>200$ units of prior U100 insulin use is increasingly used as a prerequisite to determine the appropriateness for switching a patient to U-500R. However, since prescribed total insulin dose is, in general, not available to payers in administrative databases and is not always completely collected in electronic medical records (EMR), dispensed total daily dose (dTDD), calculated on the basis of available pharmacy insulin claims, is used as a proxy for prescribed total daily dose or patients' insulin needs [20]. Derived dTDD as an insulin dose measure can be heavily influenced by patients' compliance behavior and could differ significantly from the prescribed insulin doses or patients' insulin needs.

Since the group of patients with previous U-100 dTDD $\leq 200$ units comprised a significant portion of patients prescribed U-500R, it is important to understand the implications of imposing the prerequisite of previous U-100 dTDD $>200$ units before U-500R initiation. By understanding the possible reasons for utilizing U-500R among those with U-100 dTDD $\leq 200$ units in a real-world setting, the present study hoped to shed some light on this prerequisite. This study examined patient characteristics, treatment patterns, and clinical outcomes among patients with T2D who had dTDD of $\leq 200$ units pre-U-500R initiation and $>200$ units/day post-initiation. The study comprehensively evaluated dTDD, insulin dosage in units $/ \mathrm{kg}$, adherence as reflected through proportion of days covered (PDC), and treatment fills. Key treatment outcomes included HbA1c levels, hypoglycemia, and weight gain.

\section{METHODS}

\section{Study Design}

\section{Selection Criteria, Study Cohorts, and Subgroups}

This was a retrospective cohort study using Veterans Health Administration (VHA) data from April 1, 2013 through March 31, 2018. Patients with T2D aged 18 years or over with at least one prescription claim for U-500R administration (via syringe or KwikPen) during the identification period (January 1, 2014 through June 30,2017$)$ were included. Included patients were required to have no evidence of U-500R use during the 9-month pre-index period. The first prescription claim fill date for U-500R was the index date. Patients with T2D were identified using ICD-9/10-CM codes with a validated algorithm (Table 1 in the supplementary material). Included patients were further required to have continuous health plan enrollment with medical and pharmacy benefits for at least 9 months pre- and at least 9 months post-index date, and at least one pre-index claim for any insulin other than U-500R. In addition, patients were required to have at least one HbA1c measurement within 90 days pre-index or up to 30 days post-index event and at least one additional HbA1c measurement at any time between 30 and 270 days (9 months) post-index event. Among multiple HbA1c measurements in follow-up, the latest measurement was recorded. Patients were excluded if they had evidence of pump use in the 9-month post-index period, and/or claims indicating dTDD $>2000$ units/day in the post-index period. Finally, only patients with mean dTDDs of $\leq 200$ in the pre-index and $>200$ in the post-index period were included. In addition to analyses among all U-500R initiators (irrespective of device), separate analyses were performed on syringe and KwikPen initiators cohorts with the index event defined as the first fill for syringe and KwikPen, respectively. 


\section{Study Measures}

\section{Demographic and Clinical Characteristics}

Demographic characteristics were evaluated at U-500R initiation. Clinical characteristics including Quan-Charlson comorbidity index (Q-CCI) scores, body mass index (BMI; derived from height and weight available in the data), and comorbidities were evaluated in the 9-month pre-index period. The proportions of patients prescribed at least one type of insulin pre-index were recorded.

\section{Treatment Patterns}

Treatment pattern measures were evaluated during the pre- and post-index periods. dTDD was captured through claims for a given period (pre- and post-index) and defined as the total number of (any) insulin units dispensed divided by the total unique days of supply in the period. Given that clinical prescribing accounts for body weight, insulin dosage was calculated as dTDD per kilogram of body weight (units/kg) among patients with capturable weight data in the pre- and post-index periods. In addition, both dTDD (units/day) and insulin dosage (units $/ \mathrm{kg}$ ) were calculated at the time of the first U-500R claim.

Adherence was measured using PDC [21], calculated as the percentage of unique days covered by any insulin in a 9-month period. For PDC and dTDD calculation, overlapping days were not stacked for concomitant use of different types of insulins (e.g., basal and bolus insulins), but were stacked for claims with the same type of insulin. Patients with PDC $\geq 80 \%$ were considered adherent [22-24]. Concomitant non-insulin medication usage was reported.

\section{Treatment Outcomes}

Pre-index HbA1c was measured from 90 days before the index date through 30 days after; among multiple values, the measurement closest to the index date was reported. The reported post-index HbA1c value was the latest observed between the end of the aforementioned 30-day post-index transition period and the end of follow-up.
Probable or documented symptomatic hypoglycemic events were identified using the Ginde algorithm [25, 26], presence of blood glucose $\leq 70 \mathrm{mg} / \mathrm{dl}$, or evidence of intramuscular glucagon administration. The numbers of hypoglycemic events per person per year (PPPY) were reported. Among patients with data available, body weight was evaluated. Both hypoglycemia and weight were evaluated during the pre- and post-index periods.

\section{Statistical Analysis}

All variables were first analyzed descriptively. The statistical significance of differences for categorical variables were determined with McNemar's test, and with the paired $t$ test for continuous variables. The difference in HbA1c and PDC before and after switching to U-500R was evaluated with mixed linear models (MLM) that incorporated both the fixed effects from demographics, BMI, pre-index Q-CCI score, index device (syringe/KwikPen), and the random effects of dTDD (pre/post) and time (pre/post), due to multiple assessments from the same individual. Additionally, in the model for PDC, the interaction of dTDD with time and that of BMI with the index device were included. For the HbA1c model, the index device and the interaction of race with index device were added to the aforementioned demographic and clinical characteristics. Factors associated with the number of hypoglycemia events in the preand post-periods were evaluated with a zeroinflated negative binomial mixed model (ZINBMM). Pre-index HbA1c, index device, and the interaction of dTDD with index device were added as covariates to the demographic and clinical characteristics in the ZINBMM. These analyses were repeated for the syringe and KwikPen subgroups.

\section{Compliance with Ethics Guidelines}

Since this study did not involve the collection, use, or transmittal of individually identifiable data, it was deemed exempt from institutional review board (IRB) review. Both the datasets and the security of the offices where analysis was 
completed (and where the datasets are kept) meet the requirements of the Health Insurance Portability and Accountability Act of 1996. Solutions IRB determined this study to be EXEMPT from the Office for Human Research Protections (OHRP)'s Regulations for the Protection of Human Subjects (45 CFR 46) under Exemption 4: Research involving the collection or study of existing data, documents, records, pathological specimens, or diagnostic specimens, if these sources are publicly available or if the information is recorded by the investigator in such a manner that subjects cannot be identified, directly or through identifiers linked to the subjects. The HIPAA Authorization Waiver was granted in accordance with the specifications of 45 CFR 164.512(i).

\section{RESULTS}

\section{Study Population}

Of the total $2391 \mathrm{U}-500 \mathrm{R}$ initiators who switched from U-100 to U-500R, $42 \%(N=1002)$ with preindex $\mathrm{dTDD}>200$ units/day were excluded from the study. Of the remaining 58\% $(N=1398)$ with pre-index dTDD $\leq 200$ units/day, $14 \%$ $(N=198)$ with post-index dTDD remaining at $\leq 200$ units/day were further excluded. The remaining $86 \%(N=1191)$ of $\mathrm{U}-500 \mathrm{R}$ initiators who transitioned from $\leq 200$ to $>200$ units/day dTDD after initiation were included (Fig. S1 in the supplementary material). Among patients initiating $U-500 R$ via syringe $(N=1766), 56.5 \%$ $(N=998)$ had pre-index dTDD $\leq 200$ units/day, of which $93.5 \%(N=933$; included as the syringe cohort) had dTDD $>200$ in the post-index period. Among patients initiating U-500R via KwikPen $(N=647), 62.3 \%(N=403)$ had pre-index dTDD $\leq 200$ units/day, of which $65.3 \%$ ( $N=263$; included as the KwikPen cohort) had dTDD $>200$ units/day in the post-index period (Fig. 1).

\section{Patient Characteristics}

Patient characteristics appear in Table 1 unless otherwise specified. The mean age was approx.

\section{U-500R Syringe Cohort}

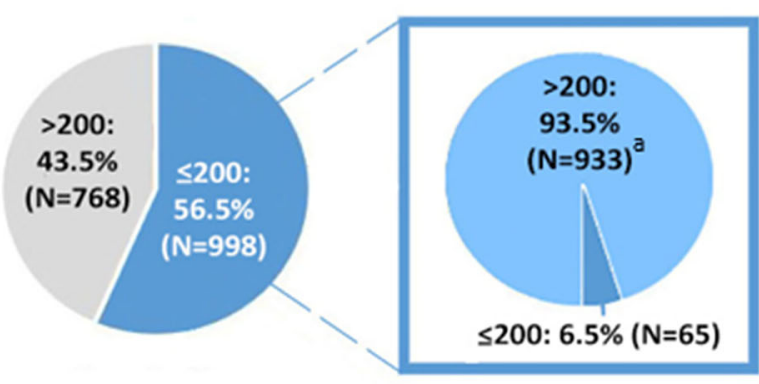

Pre-index

Post-index

\section{U-500R KwikPen Cohort}

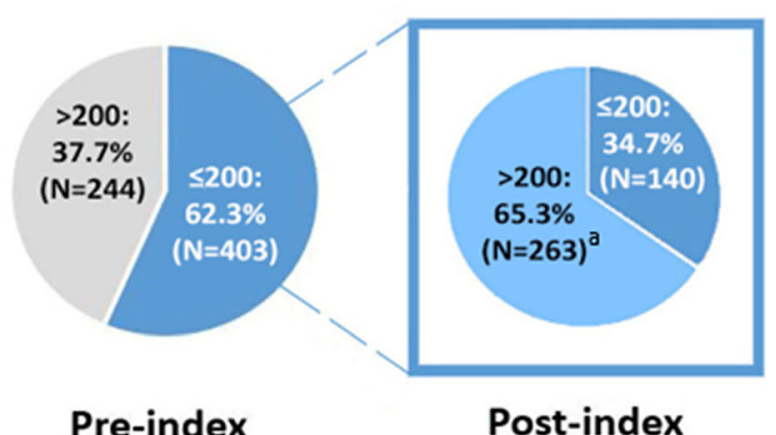

Fig. 1 dTDD distribution before and after U-500R initiation. ${ }^{\mathrm{a}}$ Patients with pre-index $\mathrm{dTDD} \leq 200$ units/day and the post-index $\mathrm{dTDD}>200$ units/day group were included in this analysis. $d T D D$ dispensed total daily dose

63 years, with predominantly male $(95.6 \%)$ and white $(82.4 \%)$ patients. The mean Q-CCI score was $3.5 \quad( \pm 2.1) \quad$ (moderate comorbidity $=2.0-4.9$ ) [27]. Among the $79.5 \%$ of patients who had BMI data, the mean BMI was 39.7 $( \pm 6.9)$. The most common comorbidity was hypertension (90.2\%) followed by neuropathy (45.9\%) and coronary artery disease $(32.0 \%)$. During the pre-index period, $86.4 \%$ of patients were prescribed more than one type of insulin, while $5.3 \%$ patients had evidence of basal insulin only. Most patients use U-500R as a monotherapy, as seen by the $78.6 \%$ who had claims for U-500R only after U-500R initiation, leaving $21.4 \%$ of patients with evidence of other insulin use (Table 2). 
Table 1 Demographic and clinical characteristics among U-500R initiators

\begin{tabular}{|c|c|c|}
\hline \multirow[b]{2}{*}{ Age (years) } & \multicolumn{2}{|c|}{$\begin{array}{l}\text { U-500R } \\
\text { initiators } \\
(N=1191)\end{array}$} \\
\hline & & \\
\hline Mean, SD & 62.77 & 8.16 \\
\hline $18-55$ & 219 & $18.39 \%$ \\
\hline $56-64$ & 368 & $30.90 \%$ \\
\hline$\geq 65$ & 604 & $50.71 \%$ \\
\hline \multicolumn{3}{|l|}{ Sex, $n(\%)$} \\
\hline Male & 1139 & $95.6 \%$ \\
\hline Female & 52 & $4.4 \%$ \\
\hline \multicolumn{3}{|l|}{ Race, $n(\%)$} \\
\hline White & 981 & $82.4 \%$ \\
\hline Black & 129 & $10.8 \%$ \\
\hline Others & 81 & $6.8 \%$ \\
\hline Body mass index, mean (SD) & 39.67 & 6.85 \\
\hline $\begin{array}{l}\text { Quan-Charlson comorbidity index } \\
\text { score, mean (SD) }\end{array}$ & 3.52 & 2.08 \\
\hline \multicolumn{3}{|l|}{ Comorbidities, $n$ (\%) } \\
\hline Retinopathy & 245 & $20.6 \%$ \\
\hline Nephropathy & 231 & $19.4 \%$ \\
\hline Neuropathy & 547 & $45.9 \%$ \\
\hline Coronary artery disease & 381 & $32.0 \%$ \\
\hline Peripheral vascular disease & 115 & $9.7 \%$ \\
\hline Congestive heart failure & 174 & $14.6 \%$ \\
\hline Hypertension & 1074 & $90.2 \%$ \\
\hline Depression & 332 & $27.9 \%$ \\
\hline Obesity & 685 & $57.5 \%$ \\
\hline Malignant tumor & 93 & $7.8 \%$ \\
\hline
\end{tabular}

\section{Treatment Patterns}

Treatment patterns appear in Table 2, unless otherwise specified. Compared to the pre-index period, the average dTDD during the 9 months post-U-500R initiation increased markedly by 199 units/day, from $147( \pm 35)$ units pre-index to $346( \pm 109)$ units post-index $(p<0.0001)$ (Fig. S3). Notably, the dTDD of the index (first) U-500R claim was $354( \pm 137)$ units (Fig. S3). Among the 1170 (98.2\%) patients with weight data, the mean insulin dosage increased considerably after initiation (from 1.2 to 2.8 units/ $\mathrm{kg}, \quad p<0.0001$ ) (Fig. 2). The proportion of patients with PDC $>80 \%$ did not increase significantly after initiation in univariate analysis; however, after adjustment for potential covariates, MLM analysis revealed a significant increase in PDC after initiation $(p<0.0001)$ (Table S2). The number of fills decreased significantly after initiation, (from $8.5[ \pm 4.6]$ to $6.5[ \pm 2.8], p<0.0001)$ as more patients were on insulin monotherapy. Before initiation, 162 patients $(13.6 \%)$ were prescribed a monotherapy, while 936 (78.60\%) were prescribed U-500R as monotherapy post-initiation.

Proportions of biguanides (59.9\% vs $53.1 \%$, $p<0.0001)$ or sulfonylureas $(12.7 \%$ vs $3.4 \%$, $p<0.0001)$ use decreased after initiation, while those of SGLT2 $(1.3 \%$ vs $3.2 \%, p=0.0004)$ or GLP-1 $(7.4 \%$ vs $12.3 \%, p<0.0001)$ use were small but increased.

Treatment patterns for syringe and KwikPen initiators were similar to the overall group, except that in the KwikPen initiators the increase in dTDD ([KwikPen: 149 units vs 284 units, $p<0.0001$ ]; [syringe: 147 units vs 363 units, $p<0.0001]$ ) and insulin dosage ([KwikPen: 1.23 units/kg vs 2.33 units $/ \mathrm{kg}, p<0.0001]$; [syringe: 1.20 units $/ \mathrm{kg}$ vs 2.92 units $/ \mathrm{kg}$, $p<0.0001])$ after initiation was lower as compared with syringe initiators (Table S3).

\section{Treatment Outcomes}

Treatment outcomes are reported in Table 2 unless otherwise specified. The mean HbA1c decreased by $1.0 \%$, from $9.6 \%( \pm 1.8 \%)$ pre-index to $8.6 \%( \pm 1.6 \%)$ in the post-index period $(p<0.0001)$. MLM results confirmed a significant decrease in HbA1c after adjusting for covariates and individual random effects $(p<0.0001 ;$ Table S4). The proportion of 
Table 2 Treatment patterns and outcomes before and after U-500R initiation

U-500R initiators $(N=1191)$

\begin{tabular}{lll}
\hline Before Initiation & $\begin{array}{l}\text { After Initiation } \\
(N=1191)\end{array}$ & $p$ value
\end{tabular}

Insulin regimen in the post-index period, $n(\%)$

U-500R monotherapy

U500R plus other insulins

Dispensed total daily dose (dTDD)

Mean (SD)

Median

Change in dTDD, mean (SD)

Index dTDD (first U-500R claim), mean (SD)

Insulin dosage in units $/ \mathrm{kg}^{\mathrm{a}}$

Mean (SD)

Median

Index insulin dosage (first U-500R claim), mean (SD)

Total Insulin units, mean (SD)

Proportion of days covered (PDC)

PDC in \%, mean (SD)

$\mathrm{PDC}>80 \%, n(\%)$

Number of fills, $n$ (\%)

Concomitant medications, $n$ (\%)

Thiazolidinediones

Sulfonylureas

Biguanides

Meglitinides

Alpha-glucosidase inhibitors

Dipeptidyl peptidase (DPP) 4 inhibitor

SGLT2 inhibitor

GLP-1 receptor agonists

Amylin agonist (pramlintide)

HbAlc

HbAlc in \%, mean (SD)

Change in $\mathrm{HbAlc} \%$, mean (SD)

$\begin{array}{ll}- & 936(78.60 \%) \\ - & 255(21.40 \%)\end{array}$

$147.2(35.1)$

346.3 (108.6)

$<0.0001$

151.4

333.3

$199.2(112.6)$

353.9 (137.5)

$1.2(0.4)$

1.2

$47,805.3(20,645.4)$

$75 \%(20)$

$576(48.4 \%)$

$8.5(4.6)$

$13(1.2 \%)$

$151(12.7 \%)$

$713(59.9 \%)$

$1(0.1 \%)$

$28(2.4 \%)$

$45(3.8 \%)$

$16(1.3 \%)$

$88(7.4 \%)$

$14(1.2 \%)$

$9.6(1.8)$
$2.8(1.0)$

$<0.0001$

2.6

$2.9(1.3)$

$91,864.7(39,189.6)<0.0001$

$76 \%(18)$

0.0695

$563(47.3 \%)$

0.5422

$6.5(2.8)$

$<0.0001$

(0.7\%)

0.1655

41 (3.4\%)

$<0.0001$

$633(53.2 \%)$

$<0.0001$

$0(0.0 \%)$

0.0004

0.0495

1.0000

0.0004

38 (3.2\%)

$<0.0001$

147 (12.3\%)

$<0.0001$

$38(3.2 \%)$

8.6 (1.6)

$<0.0001$

Hypoglycemia 
Table 2 continued

\begin{tabular}{|c|c|c|c|}
\hline & \multicolumn{3}{|c|}{ U-500R initiators $(N=1191)$} \\
\hline & $\begin{array}{l}\text { Before Initiation } \\
(N=1191)\end{array}$ & $\begin{array}{l}\text { After Initiation } \\
(N=1191)\end{array}$ & $p$ value \\
\hline Hypoglycemia, $n$ (\%) & $486(40.8 \%)$ & $676(56.8 \%)$ & $<0.0001$ \\
\hline Hypoglycemia events PPPY, mean (SD) & $2.04(4.61)$ & $3.30(5.52)$ & $<0.0001$ \\
\hline \multicolumn{4}{|l|}{ Body weight } \\
\hline $\begin{array}{l}\text { Number of patients with weight data available before and } \\
\text { after initiation, } n(\%)\end{array}$ & & $1170(98.2 \%)$ & \\
\hline Weight in $\mathrm{kg}$, mean (SD) & $126(23.9)$ & $128(24.6)$ & $<0.0001$ \\
\hline Body mass index, mean (SD) & $39.67(6.850)$ & & \\
\hline
\end{tabular}

$d T D D$ dispensed total daily dose, NIA non-insulin injectable antihyperglycemia agents, SGLT2 sodium-glucose cotransporter 2, GLP-1 glucagon-like peptide 1

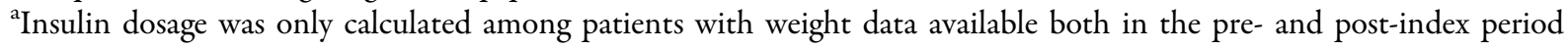

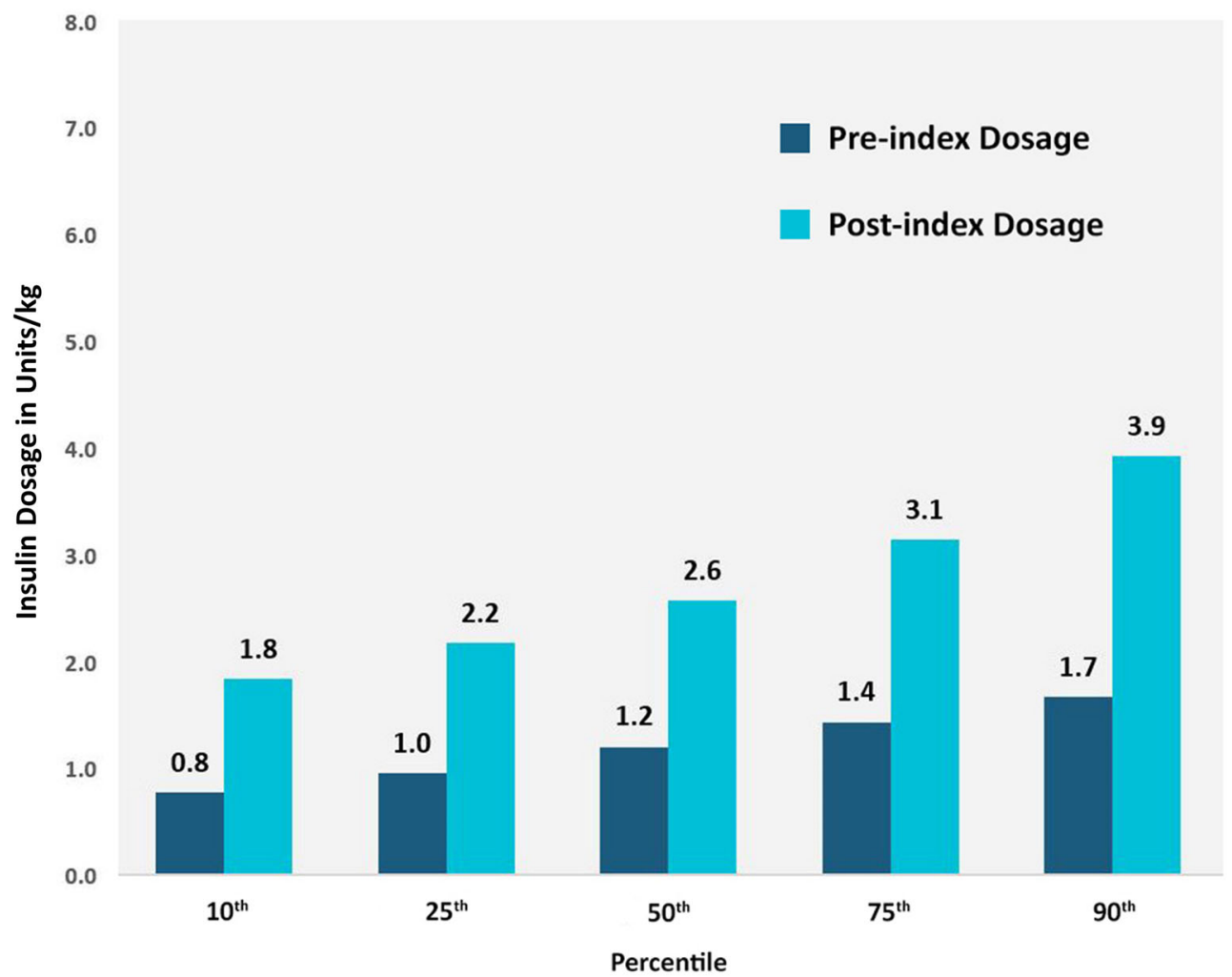

Fig. 2 Insulin dosage (units/kg) distributions before and after U-500R initiation. Pre-index and post-index dosage averaged over 9-month pre-index and 9-month post-index periods, respectively. 10th percentile (10\%): first decile;

25th percentile (25\%): first quartile (Q1); 50 th percentile (50\%): median (fifth decile/second quartile); 75 th percentile (75\%): third quartile (Q3); 90th percentile (90\%): ninth decile 


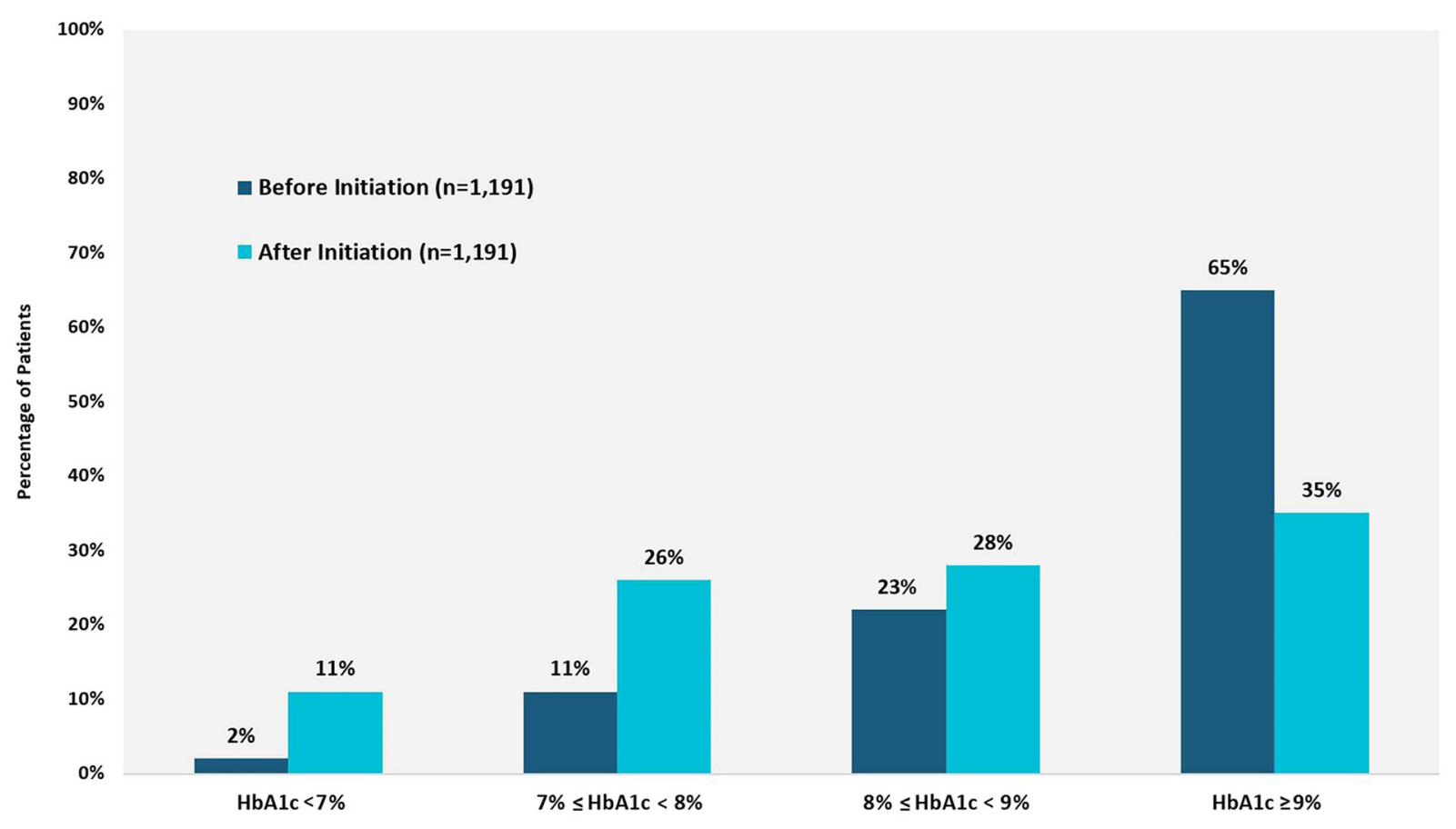

Fig. $3 \mathrm{HbA} 1 \mathrm{c}$ distributions before and after U-500R initiation

patients with $\geq 9 \%$ HbA1c dropped from $64.7 \%$ to $34.7 \%$ and the proportion with $\leq 7 \% \mathrm{HbA} 1 \mathrm{c}$ increased from $2.1 \%$ to $11.3 \%$ (Fig. 3). The symptomatic hypoglycemia event rate was estimated to be $2.0( \pm 4.6)$ events PPPY pre-index and $3.3( \pm 5.5)$ events PPPY post-index $(p<0.0001)$, and ZINBMM results showed that the post-index symptomatic hypoglycemia event incidence rate was 1.9 times greater than the pre-index incidence rate (adjusted rate ratio [aRR], 1.9; $p<0.0001$, Table S5). While the HbA1c change was mostly similar among the syringe and KwikPen initiators, the number of hypoglycemia events is notable (Table S3). Among syringe initiators, the hypoglycemia event rates pre- and post-index were $1.3( \pm 3.3)$ vs $2.8( \pm 4.8)$ events PPPY, respectively $(p<0.0001)$. Among KwikPen initiators, hypoglycemia event rates in the same periods were $4.5 \quad( \pm 7.1) \quad$ vs $5.1 \quad( \pm 7.1)$ events PPPY $(p=0.0329)$. Additionally, the mean body weight among all initiators increased from 126 $( \pm 24) \mathrm{kg}$ to $128( \pm 25) \mathrm{kg}(p<0.0001)$, with similar trends among the device subgroups. Key treatment patterns and outcomes appear in Fig. S3.

\section{DISCUSSION}

This study evaluated treatment patterns and outcomes among a subgroup of U-500R initiators who switched from filling $\leq 200$ units/day of U-100 insulins to filling $>200$ units/day after U-500R initiation, a cohort that has never been studied before. We observed that nearly twothirds of these patients had pre-index HbA1c greater than $9 \%$, and the mean HbA1c improved by $1 \%$ on average after U-500R initiation, with an unexpectedly large increase in average dTDD of 199 units. This speaks to the question of why patients who appear to be well below dosage levels of 200 units/day are prescribed U-500R in real clinical practice. The rather poor glycemic control in this group of patients while on U-100 may be largely attributable to a substantive subpopulation of patients with diabetes who are underinsulinized because of treatment compliance issues $[12,18,28]$. Compliance issues may be exacerbated among patients prescribed higher-dose U-100 regimens because of more frequent titration and glucose monitoring, the larger number of injections with multiple insulins, 
and injection site discomfort, which can lead to poor glycemic control $[12,18]$.

Other than the greater dose change magnitude, our study results align in general direction with previous clinical and observational studies of U-500R initiation, despite pre-index dosage and HbA1c differences [9, 18, 19, 29, 30]. For example, in their 2015 clinical trial, Hood et al. reported a mean TDD (confirmed dose) of 287 units (or 2.4 units $/ \mathrm{kg}$ ) with a mean HbA1c of $8.7 \%$ at baseline [3]. In a 2021 real-world study, Hood et al. reported a mean dTDD (from claims) of 249 units (or 2.0 units $/ \mathrm{kg}$ ) with a mean HbA1c of $9.3 \%$ pre-index [19]. In contrast, our study found a mean dTDD (from claims) of 147 units (or 1.2 units $/ \mathrm{kg}$ ), with a mean HbA1c of $9.6 \%$ pre-index. While the magnitude of insulin dose increases differed across these studies, remarkably, all found nearly the same mean post-index total daily dose (around 335-346 units/day [or 2.7-3.0 units/kg]) [3]. Overall, the magnitude of glycemic improvement after U-500 initiation was also similar among these studies. Our finding of a $1.0 \%$ reduction in HbA1c was slightly lower than the findings in Hood et al.'s 2015 clinical trial (TID 1.1\%, BID $1.2 \%$ ) [3], but the improvement was greater than that observed $(0.8 \%)$ among U-500 initiators in the same VA study cohort already filling claims for dTDD of $>200$ units/day before switching. These similarities in direction but discrepancies in magnitude suggest the possibility of widespread underinsulinization of patients with similar unmet insulin needs but varying severity levels.

Given the extent of the observed dose increase and HbA1c reduction, the increases in symptomatic hypoglycemia were expected and modest. The increases we observed were similar to those of Hood et al. (1.3 vs 1.0 PPPY) [19], although not directly comparable to findings in Hood et al. because of differing definitions.

The observed general trends appear to be consistent across demographic lines. Notably, patient characteristics in our study only differed substantively from those observed in Hood et al. in that the VHA patients in our study included considerably more men (95.6\% vs $52.9 \%)$ and were much older (mean age approx. 63 years vs approx. 55 years). The VHA population was also heavier (mean weight approx. $127 \mathrm{~kg}$ vs approx. $122 \mathrm{~kg}$ ) [3], yet BMI results were comparable; these differences are probably reflections of the majority of older male patients in the VHA system. Moreover, in a MarketScan data cohort study of non-elderly U-500R KwikPen initiators, a similar dose increase (from 153 units/day preindex to about 250 units/day post-U-500R initiation) was also observed [31].

The similarities between these studies suggest that underinsulinization may be common among high-dose insulin patients utilizing U-100 insulins. Most importantly, our findings of comparatively greater dose change magnitude with modest increase in symptomatic hypoglycemia suggest a subset of at-risk patients who are quite severely underinsulinized. This trend may be a reflection of patients with compliance compromised by poor disease insight, emotional issues regarding self-control and perceived stigma, adverse lifestyle factors, or low tolerance of multiple injections and associated pain and discomfort [1-5]. Given the advantages of U-500R's simplified regimen in this regard, clinicians may be prescribing U-500R for these patients in attempts to correct serious compliance issues. Although actual patient insulin needs and the extent of underdosing are not discernable in claims data, U-500R appeared to be part of an effective treatment strategy for improving glycemic control.

Hood et al.'s 2015 clinical trial and Kabul et al.'s subsequent analysis of patient-reported outcomes suggested that suboptimal insulin dosing is likely to be an issue among severely insulin resistant patients with T2D $[3,16]$. However, testing this hypothesis in the realworld setting has presented a central research challenge. Administrative claims data captures only dispensed amounts of insulins as purchased by patients. Therefore, doses calculated from medication fills in claims, represented as dTDD or insulin dosage (units/kg) in our study, do not necessarily reflect the actual amounts either needed or administered by patients. Likewise, claim fills do not necessarily reflect the actual insulin doses prescribed by physicians, as not all prescriptions are actually filled. Further, EMR databases also do not capture 
prescribed insulin doses in a manner that is feasibly observable in this type of study. The structured variable fields of available data sources are not designed in a way that allows insulin-specific information to be captured. Rather, insulin prescribing information captured in unstructured notes is rarely standardized or complete, and extraction methods such as natural language processing (NLP) have variable accuracy, reduce sample sizes precipitously, and were outside the feasibility scope of the present study. Therefore, simple observation of only the total daily dose as calculated using insulin claims does not support definitive conclusions as to whether a given patient requires or is prescribed high-dose insulin or not, unless the observed dTDD already exceeds the high-dose threshold of $>200$ units/day.

It is understandable that the alarming increase in the prevalence of obesity and a consistent increase in T2DM are causing payers to be concerned with the clinical and cost impact brought by possible increased utilization of U-500R. Such concerns may have led to imposing a prerequisite of evidence of prior U100 insulin daily dose $>200$ units. Since only dispensed total daily dose from insulin claims is available for assessing the prerequisite, our study suggested that, in a population that struggles with complex U100 basal-bolus regimen, claim-dose-based prerequisite could be counterproductive and could deny or delay high-dose insulin patients of a much-needed treatment that is effective in addressing noncompliance.

Conventional retrospective adherence measures such as PDC are limited in their ability to reflect actual compliance because of the complexity and heterogeneity of insulin treatment regimens, and they are not recommended as sole measures of insulin regimen compliance $[21,32,33]$. Separate, overlapping fills for the different U-100 basal and bolus insulins could inflate the proportions of days covered by insulins in patients' possession as compared with a monotherapy like U-500R [25]. Furthermore, PDC measure indicates whether any insulin has been used rather than whether enough insulin has been used every day. The use of PDC alone could fail to clearly illuminate suboptimal compliance patterns among this population, such as those suggested by this study. This study also reflected the ongoing struggle with diabetes management among the high-dose insulin population. Even though the 9-month use of U-500R reduced the average HbA1c levels by $1 \%$ from the dangerously high $9.6 \%$, the glucose control level is far from satisfactory. Thus, careful consideration of other factors is necessary to identify all patients with high insulin needs and compliance issues in the real-world setting.

Our study may also speak to a correction of clinician ambivalence about prescribing U-500R stemming from fears of hypoglycemic events. The results suggest such safety concerns may be disproportionate to real-world outcomes. Results did show a significant increase, but the magnitude of the increase was both in line with previous studies and expected given the clinically important decrease in HbA1c levels. Hypoglycemia events among KwikPen initiators were notable in that mean numbers were high both before and after initiation, yet the difference, while significant, was relatively modest $(4.5-5.1 ; p=0.0329)$. These results may speak to some channeling bias, i.e., clinicians may be steering patients prone to hypoglycemia toward U-500R via KwikPen. In line with precedent, U-500R delivered via syringe was prescribed mostly for patients who had much higher insulin requirements and were less prone to hypoglycemia [34].

\section{STRENGTHS AND LIMITATIONS}

This study employed a large dataset with clinical measures that supported comprehensive evaluation of treatment patterns and outcomes and provided insight into patients who transitioned from dTDD $\leq 200$ to $>200$ units/day of insulin after U-500R initiation. Moreover, high VHA medication subsidization allowed for de facto cost-neutrality that helped remove cost as a confounder.

The study had inherent limitations of all retrospective administrative database analyses, and interpretation is limited to association rather than causal inference. Because physician 
prescriptions were not capturable in the database and not all prescriptions are actually filled, noncompliance could not be confirmed by comparing dTDD with prescribed TDD. Continued titration needs after U-500R initiation [3] could also not be evaluated. The algorithm for hypoglycemia events likely captured only severe events, possibly underestimating hypoglycemia of other severities. Finally, the VHA database is composed of nearly all male, predominantly white patients, and is limited to enrollees without separate commercial coverage, all of which may limit the generalizability of the results.

\section{CONCLUSIONS}

A significant portion of U-500R initiators were found to have lower than 200 units/day insulin dose in their insulin claims and poor glycemic control while previously prescribed U-100 insulin. U-500R initiation was associated with large increases in purchased insulin dosage as well as clinically important improvement in glycemic control, together with relatively modest increases in symptomatic hypoglycemic events. This suggests U-500R addresses unmet patient insulin needs, in particular among the most markedly underdosed. As prescribed insulin dosage data is unavailable or incomplete in administrative claims data, the actual prevalence of patients requiring high dose insulin is unknown. Our findings suggest there may be more of these unrecognized high-dose patients with poor glycemic control. Imposing a claimsbased prerequisite such as prior U-100 dTDD > 200 units for U-500R qualification could have unintended consequences in this highly underinsulinized population. High-dose insulin patients can incur some of the highest health care costs among patients with diabetes, and this patient population is growing as a result of increasing obesity. Thus, this study emphasizes the importance of continued investigation of the prevalence and causes of unmet needs among high-dose insulin patients. Such research can aid in the identification of compliance issues and facilitate more effective management strategies among a vulnerable patient population that represents a growing public health concern.

\section{Acknowledgements}

Funding Sponsorship for this study and Rapid Service Fee were funded by Eli Lilly and Company.

Authorship All named authors meet the International Committee of Medical Journal Editors (ICMJE) criteria for authorship for this article, take responsibility for the integrity of the work as a whole, and have given their approval for this version to be published.

Author Contributions All authors were involved in the conception, design, analysis, and interpretation of the data, as well as the drafting and revision of the paper. All authors have approved the final version for publication and accept responsibility for all aspects of the work.

Medical Writing, Editorial, and Other Assistance The authors wish to acknowledge Ishveen Chopra for analytical assistance, and Michael Kane for medical writing assistance, all in the employ of STATinMED Research and funded by Eli Lilly and Company.

Disclosures J. Chen. L Fan, and R. Pollom are employees of Eli Lilly and Company, Indianapolis, IN. S. Borra is an employee of SIMR, LLC, Plano, TX-a paid consultant to Eli Lilly and Company, and acted as such in the conduct of this study and manuscript development. At the time of this study, A. Hong was an employee of SIMR, LLC, Plano, TX. R. Hood has received remuneration from Eli Lilly and Company, Indianapolis, IN. for consultation services and as part of their speaker bureau and also has received honoraria from Novo Nordisk, Plainsboro, NJ, as part of their speaker's bureau.

Compliance with Ethics Guidelines Since this study did not involve the collection, use, or transmittal of individually identifiable data, it was deemed exempt from institutional review board (IRB) review. Both the datasets and the security of the offices where analysis was 
completed (and where the datasets are kept) meet the requirements of the Health Insurance Portability and Accountability Act of 1996. Solutions IRB determined this study to be EXEMPT from the Office for Human Research Protections (OHRP)'s Regulations for the Protection of Human Subjects (45 CFR 46) under Exemption 4: Research involving the collection or study of existing data, documents, records, pathological specimens, or diagnostic specimens, if these sources are publicly available or if the information is recorded by the investigator in such a manner that subjects cannot be identified, directly or through identifiers linked to the subjects. The HIPAA Authorization Waiver was granted in accordance with the specifications of 45 CFR 164.512(i).

Data Availability The dataset supporting the conclusions in this article is available from the United States Department of Veterans Affairs. However, restrictions apply to the availability of these data, which were used under license for this study and are not publicly available.

\section{OPEN ACCESS}

This article is licensed under a Creative Commons Attribution-NonCommercial 4.0 International License, which permits any noncommercial use, sharing, adaptation, distribution and reproduction in any medium or format, as long as you give appropriate credit to the original author(s) and the source, provide a link to the Creative Commons licence, and indicate if changes were made. The images or other third party material in this article are included in the article's Creative Commons licence, unless indicated otherwise in a credit line to the material. If material is not included in the article's Creative Commons licence and your intended use is not permitted by statutory regulation or exceeds the permitted use, you will need to obtain permission directly from the copyright holder. To view a copy of this licence, visit http://creativecommons.org/licenses/by$\mathrm{nc} / 4.0 /$.

\section{REFERENCES}

1. Davies MJ, Gagliardino JJ, Gray LJ, Khunti K, Mohan V, Hughes R. Real-world factors affecting adherence to insulin therapy in patients with type 1 or type 2 diabetes mellitus: a systematic review. Diabet Med. 2013;30:512-24.

2. Polonsky WH, Henry RR. Poor medication adherence in type 2 diabetes: recognizing the scope of the problem and its key contributors. Patient Prefer Adherence. 2016;10:1299-307. https://doi.org/10. 2147/PPA.S106821.

3. Hood RC, Arakaki RF, Wysham C, Li YG, Settles JA, Jackson JA. Two treatment approaches for human regular U-500 insulin in patients with type 2 diabetes not achieving adequate glycemic control on high-dose U-100 insulin therapy with or without oral agents: a randomized, titration-to-target clinical trial. Endocr Pract. 2015;21:782-93.

4. Hood RC, Williams S, Taneja D, Tannock LR. Clinical efficacy and patient satisfaction with U-500 insulin use. Diabetes Res Clin Pract. 2010;88: 259-64.

5. Bulchandani DG, Konrady T, Hamburg MS. Clinical efficacy and patient satisfaction with U-500 insulin pump therapy in patients with type 2 diabetes. Endocr Pract. 2007;13:721-5.

6. Eby EL, Zagar AJ, Wang P, et al. Healthcare costs and adherence associated with human regular U-500 versus high-dose U-100 insulin in patients with diabetes. Endocr Pract. 2014;20:663-70.

7. FDA approves a dedicated syringe to be used with Humulin R U-500 insulin. U.S. Food and Drug Administration website. Last updated July 8, 2016. https://www.fda.gov/drugs/drug-safety-andavailability/fda-approves-dedicated-syringe-beused-humulin-r-u-500-insulin. Accessed May 10, 2020.

8. Humulin R U-500 KwikPen may improve glycemic control in people with severely insulin-resistant diabetes [press release]. Indianapolis: Eli Lilly and Company; January 21, 2016. https://investor.lilly. $\mathrm{com} /$ releasedetail.cfm?ReleaseID=951175. Accessed Jan 21, 2016.

9. Sze D, Goldman J. Human regular 500 units $/ \mathrm{mL}$ insulin therapy: a review of clinical evidence and new delivery options. Clin Diabetes. 2018;36(4): 319-24.

10. Humuiln R Label 2018. United States Food \& Drug Administration website. https://www.accessdata. fda.gov/drugsatfda_docs/label/2018/ 018780s126s134lbl.pdf. Accessed Aug 22, 2020. 
11. Reutrakul S, Wroblewski K, Brown RL. Clinical use of U-500 regular insulin: review and meta-analysis. J Diabetes Sci Technol. 2012;6(2):412-20.

12. Al-Qazaz HK, Sulaiman SA, Hassali MA. Diabetes knowledge, medication adherence and glycemic control among patients with type 2 diabetes. Int $\mathrm{J}$ Clin Pharm. 2011;33(6):1028-35.

13. Cochran EK, Valentine V, Samaan KH, Corey IB, Jackson JA. Practice tips and tools for the successful use of U-500 regular human insulin: the diabetes educator is key. Diabetes Educ. 2014;40:153-65.

14. de la Peña A, Ma X, Reddy S, Ovalle F, Bergenstal RM, Jackson JA. Application of PK/PD modeling and simulation to dosing regimen optimization of high-dose human regular U-500 insulin. J Diabetes Sci Technol. 2014;8:821-9.

15. Schloot NC, Hood RC, Corrigan SM, Panek RL, Heise T. Concentrated insulins in current clinical practice. Diabetes Res Clin Pract. 2019;148:93-101.

16. Kabul S, Hood RC, Duan R, DeLozier AM, Settles J. Patient-reported outcomes in transition from highdose U-100 insulin to human regular U-500 insulin in severely insulin-resistant patients with type 2 diabetes: analysis of a randomized clinical trial. Health Qual Life Outcomes. 2016;14(1):139.

17. Peng X, Chen J, Fan F, Ilag L, Ly T, Johnson J. Patient reported outcomes (PRO) in a study of human regular U-500 insulin (U-500R) delivered by continuous subcutaneous insulin infusion (CSII) or multiple daily injections (MDI) in patients with T2D. Diabetes. 2018;67(S1):967.

18. Cummings DM, Lutes L, Littlewood K, et al. Regimen-related distress, medication adherence, and glycemic control in rural African American women with type 2 diabetes mellitus. Ann Pharmacother. 2014;48(8):970-7.

19. Hood RC, Borra S, Fan L, et al. Treatment patterns and outcomes, before and after Humulin R U-500 initiation, among high-dose type 2 diabetes mellitus patients in the United States. Endocr Pract. 2021;27(8):798-806.

20. Iskandar I, Peng X, Xuanyao H, et al. The trend of high-dose insulin usage among patients with diabetes in the UK: a retrospective study. Diabetes Ther. 2018;9(6):2245-57.

21. Stolpe S, Kroes MA, Webb N, Wisniewski T. A systematic review of insulin adherence measures in patients with diabetes. J Manag Care Spec Pharm. 2016;22(11):1224-46.
22. Choudhry NK, Shrank WH, Levin RL, et al. Measuring concurrent adherence to multiple related medications. Am J Manag Care. 2009;15(7):457-64.

23. Gibson TB, Song X, Alemayehu B, et al. Cost sharing, adherence, and health outcomes in patients with diabetes. Am J Manag Care. 2010;16(8): 589-600.

24. Curtis S, Boye K, Lage M, et al. Medication adherence and improved outcomes among patients with type 2 diabetes. Am J Manag Care. 2017;23(7): e208-14.

25. Workgroup on Hypoglycemia, American Diabetes Association. Defining and reporting hypoglycemia in diabetes: a report from the American diabetes association workgroup on hypoglycemia. Diabetes Care. 2005;28(5):1245-9.

26. Ginde AA, Blanc PG, Lieberman RM, Camargo CA Jr. Validation of ICD-9-CM coding algorithm for improved identification of hypoglycemia visits. BMC Endocr Disord. 2008;8:4.

27. Huang YQ, Gou R, Diao YS, et al. Charlson comorbidity index helps predict the risk of mortality for patients with type 2 diabetic nephropathy. J Zhejiang Univ Sci B. 2014;15(1):58-66. https://doi.org/10.1631/jzus.B1300109.

28. Peyrot M, Barnett AH, Meneghini LF, SchummDraeger PM. Insulin adherence behaviours and barriers in the multinational Global Attitudes of Patients and Physicians in Insulin Therapy study. Diabet Med. 2012;5(29):682-9.

29. Ovalle F. Clinical approach to the patient with diabetes mellitus and very high insulin requirements. Diabetes Res Clin Pract. 2010;90:231-42.

30. Wysham C, Hood RC, Warren ML, Wang T, Morwick TM, Jackson JA. Effect of total daily dose on efficacy, dosing, and safety of 2 dose titration regimens of human regular U-500 insulin in severely insulin-resistant patients with type 2 diabetes. Endocr Pract. 2016;22(6):653-65.

31. Chen J, Fan L, Pollom RD, et al. Insulin-related outof-pocket pharmacy costs before and after human regular U-500 insulin initiation via insulin pen in U.S. patients with type 2 diabetes. The International Society for Pharmacoeconomics and Outcomes Research (ISPOR) Conference 2020, Orlando, May 18-20, 2020.

32. Clancy ZA. MPR and PDC: implications for interpretation of adherence research results. Value Health. 2013;16(3):A53.

33. Chun J, Strong J, Urquhart S. Insulin initiation and titration in patients with type 2 diabetes. Diabetes 
Spectr. 2019;32(2):104-11. https://doi.org/10.2337/ ds18-0005.

34. Blevins T, Lane W, Rodbard D, et al. Glucose variability and time in range in type 2 diabetes treated with U-500R by pump or injection: CGM findings from the VIVID study. Diabetes Technol Ther. 2020. https://doi.org/10.1089/dia.2020.0030. 